\title{
La réalisation du movere rhétorique dans le discours journalistique sur l'exemple des titres de la critique de cinéma
}

\section{The Realisation of the Rhetorical movere in the Journalistic Discourse on the Example of the Titles of Film Reviews}

\author{
Dominika Topa-Bryniarska [dominika.topa@us.edu.pl] \\ Uniwersytet Śląski w Katowicach, Pologne
}

\begin{abstract}
RÉSUMÉ
À l'appui d'un cadre théorique situé au carrefour de la pragmatique, de la rhétorique, de l'argumentation et de l'analyse du discours, le genre de la critique de cinéma, relativement peu exploré, est analysé en tant que discours persuasif reflétant une évaluation motivée. Létude concerne les titres de quatre-vingt-dix critiques de trois comédies françaises. Dans ce contexte, l’acte de persuasion, ancré dans la lignée perelmanienne, correspond à l'appel rhétorique et se manifeste sous un ensemble de paramètres issus de la fonction rhétorique du movere qui constitue le registre délibératif de la critique. Le rôle de ce registre est détablir une « image verbale » fondée sur des valeurs et des émotions partagées. Le destinataire est ainsi invité à coproduire le sens du discours sur la base d'une co-schématisation émotive et axiologique réalisée par des lexèmes évaluatifs et affectifs, ce qui est également typique du discours publicitaire.
\end{abstract}

\section{MotS-CLÉS}

Critique de cinéma; titre; fonction rhétorique du movere; registre délibératif; lexèmes évaluatifs ; lexèmes affectifs ; co-schématisation

\begin{abstract}
Using a theoretical framework lying at the intersection of pragmatics, rhetoric, argumentation and discourse analysis, the genre 'film review', relatively little addressed, is examined in the following paper as a persuasive discourse reflecting motivated evaluation. The study is based on the titles of ninety reviews concerning three French comedies. In such a context, the act of persuasion, anchored in the Perelmanian lineage, corresponds to the rhetorical appeal. Thus, this study concerns a set of parameters stemming from the rhetorical function of movere which constitutes the deliberative register of a film review. The role of this register is to establish a "verbal image" built on shared values and affects. As a result, the addressee is invited to co-produce the meaning of the discourse on the basis of an emotive and axiological co-schematisation realised by means of evaluative and affective terms, which is also typical of advertising discourse.
\end{abstract}

\section{KeYWORDS}

Film review; title; rhetorical function of movere; deliberative register; evaluative terms; affective terms; coschematisation

REÇU 2020-01-31; ACCEPTE 2020-06-12 


\section{Introduction}

Dans le présent travail, nous nous proposons détudier certains mécanismes discursifs dont le fonctionnement linguistique et argumentatif met au premier plan la visée pathémique de la fonction rhétorique du movere destiné à émouvoir, à toucher la sensibilité au sens aristotélicien. Cette fonction est fréquemment exploitée dans l’univers dynamique du discours médiatique d’aujourd'hui centré sur l'efficacité communicationnelle, dont la critique de cinéma. Le genre de la critique - relativement peu analysé - appartient aux discours strictement persuasifs, ce qui découle de son objectif principal que nous considérons comme une évaluation motivée. Puisque l'évaluation est inhérente à l'exercice de la critique, celle-ci, dans son dispositif global de former l'opinion, se distingue par une double finalité d'informer et de persuader, en ressemblant ainsi, à bien des égards, aux procédés utilisées dans le discours publicitaire. La critique contribue alors à susciter non seulement l'intérêt du public pour l'objet décrit - le film, mais également elle incite à prendre une décision d'aller voir ou non cet objet, en fonction de sa présentation favorable ou défavorable.

Comme nous nous intéressons principalement à la fonction persuasive du discours, notre recherche s'inscrit aussi bien dans le cadre de la linguistique fonctionnelle du discours médiatique (Stöckl 2015) que dans l'espace de l'argumentation rhétorique et de l'analyse du discours (Amossy $2015: \$ 1$ ). Nous nous référons ici au sens large de l’acte de persuader ancré dans la lignée perelmanienne (Perelman - Olbrechts-Tyteca 2008 ; Perelman 1997), où la notion d'analyse de discours correspond à l'adresse rhétorique (ang. public address) plutôt qu'à la définition foucaldienne (voir Doury - Plantin 2015). Nous adoptons également la notion de schématisation de Grize (1996 ; 1998 ; 2004) car, à l’appui de la manifestation discursive des représentations et des valeurs sollicitées qui interviennent dans l'activité de schématisation, il est possible de " faire voir quelque chose à quelqu'un » (Grize $1996: 50)^{1}$. Le discours fait ainsi état du point de vue d'un destinateur, tout en recensant des informations sur ce dernier et la façon dont il se laisse voir. Lacte de persuader s'avère ainsi une stratégie complexe visant à produire certains effets psychologiques (perlocutoires $)^{2}$ auprès du destinataire à travers les émotions et les valeurs supposées partagées. De ce fait, nous croyons que les émotions influencent, de manière constructive, le discours et ses stratégies persuasives. Ainsi, nous allons entendre par argumentation émotive celle qui est incorporée à l'argumentation axiologique (rhétorique) (voir point 3). Notre optique, située à la croisée de réflexions d'inspiration sémantique, pragmatique et rhétorique, a alors pour but de jeter un éclairage sur le rôle persuasif de la visée pathémique du movere de la critique de cinéma en contexte évaluatif et affectif. Dans ce cadre, nous voulons poursuivre deux objectifs complémentaires : d'abord, nous allons entamer une réflexion d’ordre général sur la construction discursive du dispostif persuasif dans le genre de la critique orienté vers le transfert du pathos. Ensuite, nous pensons montrer comment cette construction permet d'établir une connivence socioculturelle dans ce que - à l'instar de Grize $(1996 ; 1998 ; 2004)$ - nous voulons appeler co-schématisation émotive et axiologique pour former des représentations sur le réel. Dans ce contexte, co-schématiser veut

1 Dans l'acception de Grize (1998: 121) : «Une schématisation présente, à un interlocuteur $B$, la façon dont un locuteur $A$ voit quelque réalité, littéralement elle la lui pro-pose, elle la lui donne à voir. C’est donc une Vorstellung, une représentation $"$.

2 Nous entendons par effet perlocutoire un changement que l'émetteur veut produire « dans l'univers psychique du récepteur » (Kalisz $1993:$ 54-55). 
donc dire proposer un micro-univers, une réalité partielle que le destinataire est invité à compléter et partager pour coproduire le sens du discours. Il s'agit d' " une image verbale » construite sur l'affectivité et les valeurs supposées communes, ce qui renvoie également à la manière dont le destinateur conçoit son interaction avec le public.

Le corpus qui servira à notre démonstration regroupe quatre-vingt-dix critiques identifiées sur diverses plateformes Internet grand public ciné-publicitaires ainsi que sur les sites Internet des médias franco- et polonophones non-spécialisés. Toutes les critiques concernent trois comédies francophones populaires : Intouchables (2011) (pl. Nietykalni), Qu'est-ce qu'on a fait au Bon Dieu? (2014) (pl. Za jakie grzechy, dobry Boże?) et Le sens de la fête (pl. Nasze najlepsze wesele) qui ont été publiées après la sortie des films. ${ }^{3}$

\section{Visée pathémique dans le dispositif persuasif de la critique de cinéma}

Dans la perspective que nous adoptons, la critique de cinéma fonctionne en tant quactivité socio-langagière conçue dans une intention précise et appartenant par là à la catégorie des genres institués de mode 2 « soumis à des cahiers des charges qui définissent l'ensemble des paramètres de l'acte communicationnel (rôles des partenaires, durée, lieu, support...) " mais qui « tolèrent des écarts » (Maingueneau $2007: 33$ ). C'est effectivement dans ces « écarts » que se place la subjectivité énonciative due à la visée pathémique.

Du fait que la critique - comme genre médiatique - constitue une forme d’action discursive, elle s'inscrit aussi dans le cadre d'un contrat de communication médiatique qui impose aux locuteurs des paramètres internes et externes à remplir tels que l'identité des locuteurs et leurs domaines du savoir (Charaudeau 2004 : 164). Cest aussi dans et par ce contrat que les locuteurs effectuent leurs rôles respectifs - comme instance de production (journaliste-critique ou émetteur/destinateur) et comme instance de réception (ensemble indifférencié de spectateurs potentiels ou récepteur/destinataire). Lémetteur-critique, est - comme le dit Charaudeau $(2009: 3)$ - «dans son droit à persuader [...], à justifier et à défendre une idée ou une action [...] » puisqu'il sait davantage de choses, ce qui légitime son rôle discursif de conseiller. Le récepteur-public, de son côté, occupe une position d'instance de réception, donc celle de devoir croire (ibid.), ce qui instaure « une communication empathique qui passe directement du dire de lénonciateur au croire de lénonciataire » (Bonhomme $2018: \$ 9$ ). Aussi pouvons-nous discerner deux dimensions de la visée pathémique : celle de faire adhérer à lopinion de lémetteur (orienter des comportements) et celle d'inciter à faire (mobiliser des actions) qui fondent le registre délibératif (au sens aristotélicien) de la critique de cinéma, axé sur lacte de conseiller ou de déconseiller. Par conséquent, le genre de la critique fonctionnerait en tant que catégorie communicationnelle qui, sur le plan discursif, s'afficherait - à l'instar du discours publicitaire - non seulement comme un espace de circulation de significations et de valeurs, mais aussi comme un " prescripteur » de biens de consommation, c'est-à-dire des films à voir ou à éviter.

Vu le but majeur de la critique qui consiste à fournir une évaluation motivée pour guider le récepteur dans ses choix cinématographiques (Baud $2003: \$ 5$ ), il est possible de percevoir ce genre

3 Vu la dimension du corpus, nous avons choisi d’analyser un échantillon représentatif de trente-cinq textes dont les sources et les dates de parution (là où c'est possible) sont signalées au fur et à mesure de leur étude au point 3 du présent travail. 
de discours en termes de macroacte performatif exploitant principalement le registre délibératif qui domine dans le processus de co-schématisation émotive et axiologique. Ce registre n'est pourtant pas isolé car il renferme également des traces du registre épidictique ${ }^{4}$, basé sur léloge ou le blâme, grâce auquel lémetteur peut faire preuve de ses compétences professionnelles. Les deux registres relèvent des stratégies de captation qui, comme le note Charaudeau (2002 : 92), servent à « séduire ou persuader le partenaire de léchange communicatif de telle sorte que celui-ci entre dans l'univers de pensée qui sous-tend l'acte de communication, et partage ainsi l'intentionnalité, les valeurs et les émotions dont il est porteur ». Même s'il y des voix que le pathos s'intégre dans les schémas cognitifs et qu'il ne possède pas ainsi ses propres marques distinctives au niveau discursif (Angenot $2008: 258$ ), nous ne pouvons pas y adhérer. Nous pensons en revanche que la transposition de l'affectivité sur le plan discursif est preuve de la subjectivité énonciative. Cette subjectivité peut se manifester sous diverses formes linguistiques de type lexical, syntaxique ou sémantique. Il en résulte que le choix déléments linguistiques réalisant la visée pathémique du movere sert non seulement à faire adhérer à l’opinion présentée, mais aussi à influencer le destinataire tout en modelant sa réception du discours (Forget $2018: \$ 14$; Amossy 2012). En conséquence, la critique de cinéma, comme toute autre critique culturelle (Rieffel 2006 : 55-56), informe sur l'actualité culturelle, tout en livrant un point de vue à son sujet; en d'autres termes, elle opère sur l'activité d'analyse, d'explication et d'interprétation, d'où les trois segments principaux de sa construction: partie informative focalisée sur l'objet de discours, partie analytico-critique portant sur le contenu du discours et partie évaluative fondée sur la valorisation (Wolny-Zmorzyński - Kaliszewski Snopek - Furman 2014 : 79).

Puisque nous voulons réperer les constituants linguistiques d'une mise en discours de la visée pathémique du movere à travers la co-schématisation émotive et axiologique relevant du registre délibératif, nous allons nous focaliser sur l'un des parcours de cette co-schématisation. Il s'agira alors des procédés associés à l'argumentation émotive issue de l'intitulation interprétative et incitative de la critique de cinéma. La forme condensée de ce «micro-texte de formes ou de dimensions variables " (Vigner $1980: 1$ ), tel un slogan publicitaire, joue un rôle incontournable dans le processus de séduction par le fait de "susciter une réaction spontanée et affective chez son récepteur $[\ldots] »$ (Adam - Bonhomme $1997:$ 59-60).

\section{Argumentation émotive dans les titres de la critique de cinéma}

La fonction d'attrape-lecteurs des titres de la critique de cinéma est tributaire des contraintes liées aussi bien au contrat de communication médiatique quà la portée évaluative du genre lui-même consistant à éveiller l'intérêt du destinataire et à stimuler sa réaction à ce qu'il lit (conseiller), ce qui privilégie leffet de surpise pour inviter à la lecture de la critique. De ce fait, le titre acquiert un statut incitatif et interprétatif qui, par sa suggestivité, constitue un déterminant de valorisation appuyé sur un réseau d’association activant « l'imaginaire collectif» (Ringoot $2014: 89$ ). Dès lors, en suivant l'idée de Charaudeau (1983 : 102), il est possible de constater que le titre fonctionne de

4 Lépidictique, le délibératif et le judiciaire font partie des genres de léloquence codifiés par la rhétorique ancienne (gréco-latine). Le terme de registre se réfère ainsi au type de discours ayant une portée concrète, à savoir celle de louer/ blâmer ou conseiller/déconseiller. 
façon autonome et devient un texte à soi seul qui « constitue une mine pour qui cherche à décrire des contrats et des stratégies du discours » (Charaudeau $1983: 101$ ).

L'incitation faite au récepteur, grâce à laquelle il devrait adhérer à l'univers discursif élaboré par l'émetteur, donne lieu à l'activation de contenus sémantico-pragmatiques variés (valeurs, représentations ou connotations partagées, émotions) appartenant au registre délibératif du conseil où la visée pathémique du movere correspond précisément à l'intention délibérée de mener à une conclusion par le biais de l'argumentation émotive autorisant l'adhésion à la thèse avancée par le destinateur (faire adhérer pour inciter). Il en résulte que le discours à visée pathémique est solidaire au discours à visée argumentative dans l'acception d'Amossy (2012 : 41-44; 2015).

Largumentation émotive, sous-tendant la visée pathémique du movere, met au centre la relation entre les locuteurs où l'émotionnel est compris en termes de perception de valeurs qui aide à co-schématiser, soit à formuler le rapport avec le monde. C'est ainsi que l'argumentation émotive nest pas une communication de l'émotion mais par lémotion (Plantin 2011 : 139-141), ce qui implique un usage intentionnel et stratégique de l'affectivité, perçu en termes de langage-action du pathos aristotélicien. Une telle argumentation s'inscrit alors dans la construction discursive des émotions (Plantin 2011 : 135) en ce sens que ces émotions peuvent être créées ou maniées afin de (re)construire le réel et suspendre l'esprit critique du destinataire. L’argumentation émotive est donc seulement crédible dans un groupe socioculturel déterminé, car, à l'opposé de l'argumentation logique, elle peut être qualifiée de juste et non de vraie. Le genre de la critique de cinéma savère ainsi un lieu de rassemblement. Par conséquent, la visée pathémique du movere, abordée du point de vue de sa fonctionnalité dans l'acte de co-schématisation émotive et axiologique, se construira sur la planification d’effets escomptés et jugés « relevants et proches » du récepteur pour réduire la distance temporelle et spatiale du discours.

Comme le note Wojtak (2015 : 98), le discours médiatique se distingue par une vision du monde spécifique conçue en fonction des attentes du public et de la volonté de lémetteur dêtre lu ou regardé. C’est pourquoi la construction des émotions et des valeurs véhiculées dans la co-schématisation est destinée à susciter l'intérêt du plus grand nombre des récepteurs. Largumentation émotive a ainsi pour objectif de mettre le public dans la disposition d’assigner aux films évalués - sous l'influence du discours de lémetteur - un ensemble de spécificités tantôt positives tantôt négatives dues à la consolidation des connotations et des valorisations à l'égard de l'objet présenté (hétéro-attribution de lémotion selon Plantin 2011 : 135-141).

Tous ces procédés rhétoriques et pragmatiques ne changent pas quant à leur convention ou structure ni ne renvoient en principe à une thématique spécifique; ce qui subit alors des modifications, c'est leur contenu, adapté au public préconstruit et/ou à la spécificité de l’objet de discours. En plus, afin d'augmenter la portée persuasive de l'acte de co-schématisation, ces procédés doivent créer une sorte de complicité avec le récepteur, en transformant celui-ci (même apparemment) en partenaire dans le partage d'un discours attractif, propre à emporter l'adhésion. Le recours à l'argumentation émotive doit ainsi donner au discours le masque d'une communication plus

5 Le terme de visée est compris ici en tant qu'entreprise de persuasion ou visée argumentative, ce qui loppose au discours à dimension argumentative dont le but principal est autre que la persuasion, celle-ci nétant ni directe ni avouée (voir Amossy 2015). 
personnalisée et fondée sur une relation de confiance, ce qui est certes discutable puisque toute la communication reste sous le contrôle de l'émetteur?

Puisque nous nous intéressons aux effets possibles de l'argumentation émotive, nous nallons pas spéculer à propos de ce que bel et bien ressent le destinataire, mais nous allons nous pencher sur les constituants discursifs d'une telle argumentation. Dans ce contexte, nous avons discerné deux groupes de constituants lexico-syntaxiques et sémantico-pragmatiques réalisant la visée pathémique du movere à travers l'acte de co-schématisation émotive et axiologique, ce qui contribue également à l'intensification du discours ${ }^{8}$. Lanalyse effectuée n’est que préliminaire vu la diversité des moyens qui s'inscrivent dans la pathémisation du discours.

\subsection{Lexies évaluatives à charge axiologique}

Le rôle de ces vocables, issus aussi bien de l'usage conventionnel que contextuel de la langue, est double. Tout d'abord, ils permettent de créer une impression d'univocité et d'intelligibilité en vue de présenter le film sous un jour positif (lexies mélioratives) ou négatif (lexies dépréciatives) comme le montrent les extraits qui suivent:

1) Le retour de la comédie grinçante (Cine-loisirs.fr, 17 avril 2014, consulté le 17 juillet 2019)

2) Un film au rythme infernal (Le Journal du Dimanche, 13 avril 2014)

3) Faut pas prendre les enfants du bon Dieu pour des racistes (Le Monde, 4 mai 2014)

4) Un film à l'humour lourd et potache, sans aucune finesse (Oblikon.net, sans date, consulté le 19 juillet 2019)

5) «Le Sens de la fête »: Nakache et Toledano en moyenne forme (LesEchos.fr, 3 octobre 2017, consulté le 18 juillet 2019)

6) Qu'est-ce qu'on a fait au Bon Dieu : les raisons d'un triomphe (Le Figaro, sans date)

7) Appétissant (Filmosaure.com, 4 octobre 2017, consulté le 18.07.2019)

8) Un savoureux bal des ratés - Le sens de la fête. (Mondocine.net, sans date, consulté le 19 juillet 2019)

9) "Nasze najlepsze wesele ». Dobra francuska komedia. Nareszcie. [fr. "Le Sens de la fête» Une bonne comédie française. Enfin.] (Gazeta Wyborcza, 17 mai 2018)

10) Twórcy "Nietykalnych" w znakomitej formie. [fr. Les réalisateurs d'Intouchables en forme splendide] (Niezalezna.pl, sans date, consulté le 20 juillet 2019)

11) Siła przyjaźni. « Nietykalni » [fr. La force de l’amitié] (Coolturalni24.pl, 10 avril 2012, consulté le 20 juillet 2019)

12) «Nietykalni »: bajka na faktach [fr. "Intouchables »: une fable inspirée de faits réels] (Onet.pl, 22 mars 2012, consulté le 19 juillet 2019)

13) Front weselny [fr. Le front nuptial] (Filmweb.pl, 20 mai 2018, consulté le 20 juillet 2019)

7 Ce contrôle est typique dans les genres du commentaire, car - vu leur fonction persuasive dominante - lémetteur se trouve tout naturellement placé en position privilégiée par rapport au récepteur, ce qui se reflète entre autres dans un choix subjectif d'informations à transmettre.

8 Nous nous inspirons ici de l’approche de Kerbrat-Orecchioni (1999 : 102-103) sur la subjectivité des adjectifs. 
Ensuite, les lexies évaluatives à charge axiologique, grâce à leur forte qualification attributive, sont susceptibles d'intensifier le discours sous forme amplifiante d'hyperbole méliorative ${ }^{9}$ servant à valoriser le film présenté en tant que représentant illustre du genre :

14) «Qu'est-ce qu’on a fait au Bon Dieu » : La comédie Française du printemps (Cine-buzz.fr, 15 avril 2014, consulté le 18 juillet 2019)

15) Le sens de la fête, dernière fable de l'incroyable duo français (Cineseries.fr, 4 octobre 2017, consulté le 18 juillet 2019)

16) "Le Sens de la fête » - la critique de la comédie irrésistible de l’automne (Paris Match, 4 octobre 2017)

17) Pourquoi "Le Sens de la fête » est le remède idéal au spleen de l'automne (Le Point, 4 octobre 2017)

18) Et les acteurs régalent, chacun dans son registre de prédilection, poussé au maximum [...] (Telerama, 3 octobre 2017)

19) "Intouchables»: le phénomène culturel des Français (Cinemovies.fr, 23 décembre 2011, consulté le 18 juillet 2019)

20) «Intouchables" Véritable film phénomène en France, où il a attiré plus de 19 millions de spectateurs (La Presse, 13 avril 2012)

21) «Intouchables » : irrésistible tandem (La Presse, 13 avril 2012)

22) "Intouchables": Retour sur le film de l’année (Moustique, 27 décembre 2011)

23) "Intouchables" : la comédie irrésistible d'Eric Toledano et Olivier Nakache (Toutlecine.com, sans date, consulté le 20 juillet 2019)

24) «Nietykalni »-francuska komedia wszech czasów [fr. "Intouchables »-la comédie française de tous les temps] (Telemagazyn, sans date)

25) “Nasze najlepsze wesele»-W najlepszym stylu "feelgood movie” [fr. "Le sens de la fête » : dans le meilleur style $d u$ "feelgood movie »] (Kino.org.pl, sans date)

Dans les extraits analysés, lémetteur-critique tente de projeter son enthousiasme ou sa déception sur le public-spectateur (hétéro-attribution de l'émotion) à l'aide d'un discours dynamique et mobilisant qui frappe les esprits et suscite l'intérêt chez son public. Un tel procédé consiste au fait de ne pas se contenter de présenter l'essentiel de son discours - les arguments, mais de l'intensifier dans le but de rendre ce discours plus attractif, plus facile à mémoriser et, par là, plus efficace. Ce discours incitatif, censé partagé avec le récepteur, est construit au moyen des adjectifs évaluatifs et des substantifs abstraits ou leur syntagmes (positifs ou négatifs) parmi lesquels nous avons encore distingué un groupe de syntagmes hyperboliques mélioratifs articulés autour du lieu rhétorique de "l'unique ». Le rôle de ce lieu est d'indiquer une portée maximale de l'objet de discours pour mettre le public dans la disposition d’assigner au film décrit un ensemble de spécificités totalisantes et exclusives. Il en va de même dans le discours publicitaire, contenant des adjectifs maximisants qui servent à dynamiser et à intensifier la description de l'objet de discours (produit vanté) qui doit être considéré comme le meilleur exemplaire de sa catégorie. L'utilisation des lexies évaluatives permet alors de convertir le film en un espace axiologisé, susceptible d'activer diverses connotations, valeurs et émotions - respectivement positives ou négatives. 
Une telle démarche correspond à l'insertion des traces de la subjectivité dans le discours qui, dans le contexte des extraits étudiés, prend la forme d'une modalisation intensificatrice relative à des modifications que l'on observe dans un discours de type rhétorique. Celui-ci a pour visée générale d'agir sur le récepteur, l'inciter à passer à l'action, ou au moins, créer une disposition générale à l'action. La spécificité du discours rhétorique réside alors dans son double objectif découlant des liens mutuels entre logos, pathos et ethos : on cherche à séduire et émouvoir afin de mieux persuader. Cet acte de persuader s'exerce aussi par l'image de lémetteur et par l'image qu'il se crée de son récepteur, ce qui concoure à la crédibilité de l’opinion présentée. Il en résulte que le discours rhétorique représente un cadre communicationnel dans lequel s'effectue un échange visant à faire adhérer le destinataire à une thèse ou une façon de voir, croire, faire autrement. Ainsi, les adjectifs qualificatifs, destinés à attribuer des propriétés aux substantifs, acquièrent, par cette extension rhétorique, une fonction de plus qui consiste à intensifier le discours afin d'attirer plus d'attention de la part du public. Les substantifs abstraits sont utilisés dans le même but d'intensification. Par conséquent, tous ces adjectifs et substantifs servent à rendre le discours plus vivant et plus persuasif, car ils permettent de traduire l'attitude de l'émetteur, ses jugements et ses sentiments.

De ce fait, en présentant les films évalués sous un jour favorable dans (1)-(3) et (6) - (13) - voire même très favorable, hyperbolique dans (14)-(25) - ou sous un jour défavorable dans (4)-(5), le destinateur persuade à l'appui d'une co-schématisation émotive et axiologique relevant d'un registre clairement délibératif, ce qui contribue à suggérer que d’autres comédies françaises n’ont pas eu tant de succès ou, au contraire, qu'elles étaient plus réussies. Il en résulte que, lors de l'interprétation, les lexies évaluatives (mélioratives ou dépréciatives) aboutissent à obtenir des effets de sens contextuels et persuasifs à la fois. Ces effets sont particulièrement visibles dans les extraits (1)-(3), où les lexies grinçant, infernal et raciste ont certes un premier sens négatif, mais dans le contexte du titre, elles acquièrent un signe de valeur positif, ce qui contribue à construire une image favorable du film évalué. De ce fait, par le biais de l’acte discursif déloge ou de critique, lémetteur contribue non seulement à intensifier son discours, mais aussi à construire la hiérarchie préférentielle des valeurs afin de façonner l'attitude du récepteur à l'égard du film en persuadant de le regarder ou non. Aussi, en tant que conseiller du public-spectateur potentiel, lémetteur peut-il également établir une relation de confiance et d'identification avec le récepteur pour maintenir son intérêt et l'engagement dans la communication et pour créer l'impression que les opinions exprimées dans le discours sont aussi les siens.

Si alors le film est décrit à l'aide des lexies évaluatives à charge axiologique négative telles que potache, lourd, sans aucune finesse, positive comme appétissant, savoureux, triomphe, znakomity (fr. splendide), dobry (fr. bon) ou encore hyperbolique comme irrésistible tandem, remède idéal, incroyable duo, phénomène culturel des Français, w najlepszym stylu (fr. dans le meilleur du style), francuska komedia wszech czasów (fr. la comédie française de tous les temps), cela veut dire que l'émetteur essaie de persuader par un jugement de valeur porté sur le film présenté de même que par l'apport émotionnel et connotatif que les lexies employées peuvent véhiculer. Le choix d'une lexie dépend de l'impression et du sentiment que l'émetteur souhaite laisser chez son public. Un tel discours se distingue donc par une axiologie co-schématisée qui influe sur la réception du message en faveur du film ou contre lui. C'est ainsi que lémetteur arrive à obtenir une suggestivité persuasive dans la présentation du film qui consiste à prétendre qu'on exprime ses vrais désirs et sentiments et quon s'engage dans la communication, ce qui donne une force persuasive qui va marquer 
les esprits par une description séduisante. Dans une telle optique, il serait loisible de parler d’une argumentation émotive du type impressif qui - contrairement à l'argumentation démonstrative se basant sur l'exposition et l'étayage des données (Zarefsky 2019 : 3-4) - impose péremptoirement des transferts référentiels orientés selon le choix axiologique du destinateur : le film est bon, voire exceptionnel/ le film est médiocre ou nul. Cette pathémisation du registre délibératif de la critique de cinéma, qui marque l'enthousiasme ou le découragement, contribue à produire un effet rhétorique de dynamisation de la réalité afin d'attirer l'attention du récepteur sur l'objet de discours tout en exposant et en légitimant la position de l'émetteur-conseiller.

\subsection{Lexies affectives directes et indirectes}

Ce deuxième type de vocables a pour fonction de fortifier la co-schématisation émotive et axiologique en incitant le récepteur à ressentir les mêmes émotions que l'émetteur. En conséquence, il est possible - à l'instar du discours publicitaire - de consolider l'attitude du public à l'égard du film évalué par le fait de renchérir sur l'impact de ce dernier ainsi que d’en anticiper des effets psychologiques (perlocutoires) à produire auprès du récepteur. Une telle modalisation intensificatrice du discours se traduit par la portée expressive des termes (noms, verbes et adjectifs en particulier) qui peuvent communiquer l'émotion de façon explicite (lexies affectives directes) ou implicite (lexies affectives indirectes).

Quant aux lexies affectives directes, elles reflètent fidèlement et sans contexte la réaction émotionnelle (attitude individuelle) du récepteur par rapport au film décrit. Cet engagament émotionnel se construit discursivement par l’emploi de désignations explicites renvoyant à l'état émotionnel de l'émetteur dans (26)-(30) et (34) ou par l'usage de termes qui font penser à cet état, donc qui l'incarnent ou le montrent ${ }^{10}$ comme dans (31)-(33) :

26) "Le Sens de la fête ": panique au mariage (Le Parisien, 4 octobre 2017)

27) "Intouchables": pourquoi je déteste ce film et son succès (Le Plus/Le Nouvel Observateur, 5 novembre 2011)

28) J'ai aimé "Intouchables", et alors? (Libération, 5 décembre 2011)

29) Oui, je le veux (Cinoche.com, 9 septembre 2017, consulté le 20 juillet 2019)

30) Intouchables: Omar, m'a tué (Nice Matin, 2 novembre 2011)

31) "Qu'est-ce qu’on a fait au Bon Dieu » : une comédie drôlissime réussie (Mcetv.fr, 11 mars 2014)

32) «Intouchables » et hilarants (Le Figaro, 2 novembre 2011)

33) "Intouchables», le film qui va toucher le public (Le Parisien, 27 septembre 2011)

34) Komedia "Za jakie grzechy, dobry Boże " Kochajmy się - mimo wszystko [fr. Aimons-nous malgré tout] (Gazeta Wyborcza, sans date)

En ce qui concerne les lexies affectives indirectes, elle sont destinées à décrire l'émotion en contexte, c'est-à-dire en la suggérant par voie de métonymie l'effet pour la cause où l'on procède à

10 Voir sur ce sujet Micheli (2008: \$18-19) et la distinction de Kerbrat-Orecchioni (2000:61) entre émotions dénotées et connotées. 
la description verbale d'une réaction émotionnelle ou d'un trait comportemental (geste, mimique, mouvement, posture) conventionnellement associés à une disposition affective donnée ${ }^{11}$ comme l'illustrent les extraits suivants :

35) "Le Sens de la fête », le rire au rendez-vous (Actu.fr, 13 octobre 2017, consulté le 18 juillet 2019)

36) «Le Sens de la fête » la critique: faites tourner les serviettes pour la meilleure comédie française de l'année! (Lci.fr, 3 octobre 2017, consulté le 20 juillet 2019)

37) "Qu'est-ce qu'on a fait au Bon Dieu »: Bourgeois au bord de la crise de nerfs, (Metronews.fr, 15 avril 2014, consulté le 19 juillet 2019)

38) Vous voilà cordialement conviés à l'union du rire et de l'intelligence, sur l'autel d'une divine comédie (Avoir-alire.com, 4 octobre 2017, consulté le 18 juillet 2019)

39) Dopóki śmiech was nie rozłaczy. [fr. Tant que le rire ne vous sépare pas] (Niezalezna.pl, sans date, consulté le 20 juillet 2019)

40) Cała Francja się śmieje (Polityka, 11 avril 2012) [fr. Toute la France rit]

41) «Za jakie grzechy, dobry Boże ». Najlepiej uczyć się tolerancji... płacząc ze śmiechu (Telemagazyn, 27 février 2018) [fr. Il vaut mieux d’apprendre la tolérance en pleurant de rire]

Dans les extraits ci-dessus, la visée pathémique du movere dans le registre délibératif de la critique correspond à la mise en discours du débordement émotionnel que l'émetteur tente d'inspirer vis-à-vis du récepteur pour susciter des réactions plus immédiates, orienter des comportements, ou tout simplement faire adhérer à l'opinion présentée. Largumentation émotive fait ainsi appel aux sentiments et aux désirs du public, ce qui lui confère un pouvoir incontournable dans l'acte de persuasion. C'est ainsi que l'émetteur espère s'attirer l'assentiment de la part du public, parfois même en amplifiant et en dramatisant le discours pour faciliter sa mémorisation.-

Le destinateur, par sa prestation discursive, cherche à provoquer des émotions voulues chez le public telles que la connivence et l'enthousiasme dans (29)-(41) ou le découragement et l'indifférence dans (26)-(27). Les émotions concernées peuvent être d'une part facilement décelables, transmises par l'émetteur sous forme d'émotions que lui-même éprouve, ou, du moins, feint d'éprouver : c'est le cas des extraits (26)-(34) parmi lesquels, comme dans (27)-(30), l'engagement affectif de la part du destinateur est accompagné d'une forte implication personnelle dans le discours (emploi de la première personne du singulier). D’autre part, les émotions peuvent être voilées et incorporées de manière plus subtile dans le discours, c'est-à-dire déclenchées par son contenu et par ce qu'il véhicule comme le montrent les extraits (35)-(41). Même si l'émetteur n'est pas physiquement présent pour partager ses émotions avec le public, ce partage est pourtant permis par la description de ses états internes suscités pendant et après l'expérience de la projection du film évalué. Ainsi, dans le domaine du cinéma, le partage émotionnel peut se réaliser dans un seul acte de lecture. Le récepteur-lecteur est en effet en mesure de s'identifier aux propos de l'émetteur-critique : il pourra y reconnaître les émotions qu'il a lui-même ressenti au cours de la projection du film ou qu'il pourrait éventuellement éprouver s'il décidait d'aller voir le film.

11 Voir aussi Plantin (2011 : 143-144) et ses remarques sur les signaux aval de l'affectivité. 
Cette mise en scène des émotions sollicitées soit positives, soit négatives se traduit alors par l'ethos $^{12} \mathrm{du}$ destinateur qui - comme cela a déjà eu lieu dans le cas des lexies évaluatives à charge axiologique - prétend jouer le rôle d’expert et de conseiller engagé dans la communication, se solidarisant avec d'autres spectateurs, ce qui permet par ailleurs d'orienter l'évaluation du film vers les valeurs prônées par l'émetteur dans le cadre d'une co-schématisation aménée par une axiologie positive ou négative. Ainsi, dans la construction discursive des émotions, l'émetteur suit deux voies grâce auxquelles il peut activer la relation d'identification entraînant l'implication ou l'inscription du destinataire dans le discours : d'un côté, il s'agit de créer un sentiment de proximité en raccourcissant la distance en communication par l'évocation des émotions censées partagées ; de l'autre côté, il est question de persuader le plus grand nombre possible de récepteurs. Aussi, à l'aide des lexies affectives, l'émetteur recourt-il aux arguments de communauté dits ad populum (voir Woods - Walton 1992 ; Szymanek 2012 : 59-60 ; Breton 2003 : 69) pour relater sa propre expérience de spectateur et la faire partager avec le public, ce qui en soi est plus persuasivement efficace qu'une simple voix de journaliste. Celui-ci ne doit pas partager la responsabilité de ses avis ni assumer à lui seul le discours : il est ainsi perçu comme un membre participant activement à une réflexion commune, ce qui crédibilise non seulement son ethos d'expert et de conseiller, mais aussi sollicite la mobilisation du récepteur qui entre dans un univers discursif co-schématisée, étant le lieu du partage d'émotions et de valeurs.

Une telle démarche constitue effectivement des arguments pour générer de l'émotion à travers des procédés de pathémisation dont le but est de provoquer une réaction à l'égard des propos tenus suivant la tendance générale à croire ce qui nous touche. L'utilisation des lexies affectives permet ainsi d'intensifier le discours en influant sur la sensibilité du récepteur-spectateur pour neutraliser ou bien suspendre son activité rationnelle d'analyse, ce qui fait de ces lexies de vrais arguments par l'émotion susceptibles de provoquer une adhésion presque inconditionnée à l'attitude de l'émetteur. C’est pour cette raison que les émotions font partie du processus de compréhension qui imite à bien des égards les techniques d'amalgame référentiel du discours publicitaire où l'annonceur cherche à incorporer le produit vanté dans les réactions de son utilisateur potentiel afin de favoriser une identification propice à l'efficacité de la persuasion. L'incitation faite au public à s'identifier aux émotions de l'émetteur-spectateur donne également lieu à des structures plus explicites appartenant au registre délibératif du conseil. Ainsi, cette incitation devient plus pressante dans l'emploi des modalités sollicitatives dans les extraits (38)-(39) et des modalités directives dans (34) et (36), toutes deux associées aux pôles allocutifs vous et nous. Un tel procédé aboutit à créer une sorte de complicité et de connivence avec le public, car l'emploi des modalités permet d'inclure subrepticement le récepteur dans l'expression du jugement de lémetteur et de le traiter en partenaire dans ce partage d'un discours mobilisant, propre à emporter l'adhésion. Le recours aux lexies affectives et évaluatives s'inscrit de ce fait pleinement dans la visée pathémique du movere qui sous-tend le registre délibératif de la critique de cinéma : c’est par ces stratégies de captation émotive que l’on peut attirer l'attention du public, qui - inéluctablement touché - se plonge plus volontiers dans la lecture de la critique, ce qui prouve l'efficacité de la persuasion adoptée.

12 Comme le remarque Plantin cité par Charaudeau et Maingueneau (2002: 424) : «le travail émotionnel [ou pathos] s'appuie sur le travail de l'ethos, qui en quelque sorte prépare le terrain ». 


\section{En guise de conclusion}

L'intitulation dans la critique de cinéma s'affiche principalement comme un terrain de circulation de mécanismes pathémiques dus au procédé de modalisation intensificatrice du discours. Dans ce qui est la couche proprement linguistique de cette modalisation, il est loisible d'envisager deux types de lexies, évaluatives et affectives, par lesquelles - à travers la fonction persuasive du langage - le destinateur manifeste son état émotionnel pour agir sur le récepteur et emporter affectivement son adhésion à l'appui de la visée pathémique du movere. Bien sûr, des mécanismes variés peuvent se trouver simultanément à l'œuvre dans une même critique, mais, dès le choix des titres, la visée pathémique du genre se construit à partir d'un cadre argumentatif dans lequel l'émetteur prend position et cherche à construire une communauté discursive d'appartenance en misant sur des liens interlocutifs propices à la persuasion. Ces liens correspondent au partage émotionnel et à l'emploi d'hyperboles activés dans le cadre de la co-schématisation, ce qui sert également à réaliser le but majeur de la critique qui est celui de persuader ou de dissuader. Aussi le discours va-t-il s'articuler autour d'un système de représentations, connotations et valeurs supposées communes entre les locuteurs. Le discours de la critique s'avère dans ce contexte un lieu de rassemblement émergeant du registre délibératif de la critique de cinéma qui rend possible la création de l'image de l'émetteur en tant que destinateur engagé dans la communication qui essaie délaborer une relation d'identité et de confiance grâce à laquelle le récepteur peut être traité en partenaire. Le destinataire participe donc à la construction du discours dans la mesure où les émotions et les valeurs sollicitées sont censées " résonner » chez lui pour faciliter son implication dans l'univers discursif créé par l'émetteur et réduire ainsi la distance temporelle et spatiale du discours, ce qui est aussi caractéristique du discours publicitaire. Par conséquent, la visée pathémique du movere abordée du point de vue de sa fonctionnalité - se construit sur la planification d'effets persuasifs escomptés ayant trait aux aspects socio-discursifs de l’argumentation émotive (voir aussi Amossy 2015).

Dans un tel cadre, le caractère persuasif de la critique de cinéma peut être considéré comme un trait pragmatique du genre tandis que la suggestivité et la subjectivité en constitueront un trait rhétorique. Ces spécificités de la critique aident à modeler la perception et l'opinion en suggérant le choix de telle façon que le public ait l'impression de se persuader lui-même et de prendre luimême ses propres décisions. C'est dans ce sens que la critique peut s'avérer manipulatrice. En conséquence, l'argumentation émotive sert principalement à attirer et maintenir l'attention du destinataire qui, à l’ère du pluralisme actuel des médias, est très dispersée, ce qui pourrait expliquer le double objectif de la critique de cinéma contemporaine : susciter l'intérêt pour informer et informer pour susciter l'intérêt.

Il est encore à noter que les constituants de l'argumentation émotive tels que les lexies évaluatives et affectives résultent des emprunts croisés entre le discours publicitaire et médiatique et que leur rôle est de mettre le public dans la disposition à s'engager dans le jeu interprétatif élaboré par le destinateur. Sur ce plan donc, la visée pathémique qui oriente le registre délibératif de la critique de cinéma vers une panoplie de paramètres régissant l'attractivité du message, perpétuerait à sa façon les deux grands préceptes constitutifs de la rhétorique latine - le movere et le delectare. Pour cette raison, il paraît justifié d’approfondir notre étude sur le phénomène de l'hétérogénéité pragmatico-rhétorique de la visée pathémique qui consiste à persuader non par l'argumentation démonstrative basée sur le logos dans l’analyse du film, mais par les arguments du type 
« impressionnant », relevant de la précellence du pathos sur le logos, comme p.ex. une possible sur-représentation des structures superlatives (aussi bien euphoriques que dysphoriques) dans les titres. Une telle problématique nous semble particulièrement utile et pertinente dans l'analyse de la typologie des genres du commentaire ainsi que dans la description des contrats de communication médiatique contemporains. Nous croyons que cette optique nous permettrait dévaluer si ces contrats sont bien observés et à quel point ils sassimilent à d'autres contrats, en particulier à ceux de la communication publicitaire.

\section{Références bibliographiques}

Adam, J.-M. ; \& Bonhomme, M. (1997). L’argumentation publicitaire. Rhétorique de l'éloge et de la persuasion. Paris : Nathan.

Amossy, R. (2012). L’argumentation dans le discours. Nouvelle présentation. $3^{e}$ éd. Paris : Armand Colin. . (2015). Quelle vocation empirique pour l'argumentation dans le discours ? Argumentation et Analyse du Discours, 15. doi : 10.4000/aad.2059 [Dernière consultation en septembre 2019]

Angenot, M. (2008). Dialogue de sourds. Traité de rhétorique antilogique. Paris : Éditions Mille et une nuits.

Baud, D. (2008). Analyse de genre : la critique de cinéma dans la presse quotidienne britannique. ASp. La revue du Geras, 39-40. doi : 10.4000/asp.1282 [Dernière consultation en septembre 2019]

Bonhomme, M. (2018). Modalités et stratégies rhétoriques de léloge dans le discours publicitaire. Exercices de rhétorique, 11. <http://journals.openedition.org/rhetorique/681> [Dernière consultation en octobre 2019]

Breton, P. (2003). L'argumentation dans la communication. $3^{e}$ éd. Paris : La Découverte.

Charaudeau, P. (1983). Langage et discours. Eléments de sémiolinguistique (théorie et pratique). Paris : Classiques Hachette.

. (2004). Comment le langage se noue à l'action dans un modèle socio-communicationnel du discours. De laction au pouvoir. Cahiers de Linguistique Française, 26, 151-175.

. (2009). Le discours de manipulation entre persuasion et influence sociale. Patrick Charaudeau - Livres, articles, publications. < http://www.patrick charaudeau.com/IMG/pdf/Discours_Manipulation-_ Texte_Lyon_.pdf $>$ [Dernière consultation en novembre 2019]

Charaudeau, P. ; \& Maingueneau, D. (2002). Dictionnaire d'Analyse du Discours. Paris : Seuil.

Doury, M. ; \& Plantin, Ch. (2015). Une approche langagière et interactionnelle de l'argumentation. Argumentation et Analyse du Discours, 15. doi: 10.4000/aad.2006 [Dernière consultation en octobre 2019]

Forget, D. (2016). Le pathos mis à contribution dans les discours à grande circulation. Corela, 19. doi : 10.4000/corela.4434 [Dernière consultation en septembre 2019]

Grize, J.-B. (1996). Logique naturelle et communication. Paris : PUF.

. (1998). Logique naturelle, activité de schématisation et concept de représentation. Cahiers de praxématique, 31, 115-125.

. (2004). Le point de vue de la logique naturelle. In M. Doury, \& S. Moirand (Éds.), L'Argumentation aujourd'hui. Positions théoriques en confrontation (pp. 35-44). Paris: Presses Sorbonne Nouvelle.

Kalisz, R. (1993). Pragmatyka językowa. Gdańsk: Wydawnictwo Uniwersytetu Gdańskiego.

Kerbrat-Orecchioni, C. (1999). L'énonciation. De la subjectivité dans le langage. Paris : Arman Collin. 
- (2000). Quelle place pour les émotions dans la linguistique du XXe siècle ? Remarques et aperçus. In Ch. Plantin (Éd.), Les émotions dans les interactions (pp. 33-74). Lyon : PUL.

Macagno, F.; \& Walton, D. N. (2014). Emotive Language in Argumentation. Cambridge : Cambridge University Press.

Maingueneau, D. (2007). Genres de discours et modes de généricité. Le Français aujourd'hui, 4, 27, 29-35. doi : $10.3917 /$ lfa.159.0029

Micheli, R. (2008). Lanalyse argumentative en diachronie : le pathos dans les débats parlementaires sur l'abolition de la peine de mort. Argumentation et Analyse du Discours, 1. doi: 10.4000/aad.482 [Dernière consultation en septembre 2019]

Perelman, Ch. (1997). Lempire rhétorique. Rhétorique et argumentation. $2^{\mathrm{e}}$ éd. Paris : Vrin.

Perelman, Ch. ; \& Olbrechts-Tyteca, L. (2008). Traitré de largumentation. La nouvelle rhétorique. $6^{\mathrm{e}}$ éd. Bruxelles ; Éditions de l'Universite de Bruxelles.

Plantin, Ch. (2011). Les bonnes raisons des émotions. Principes et méthode pour létude du discours émotionné. Berne : Peter Lang.

Rieffel, R. (2006). Lévolution du positionnement intellectuel de la critique culturelle. Quaderni, 60, 55-64. Ringoot, R. (2014). Analyser le discours de presse. Paris : Armand Collin.

Stöckl, H. (2015). Mediolingwistyka. O statusie i metodach (nadal) konstytuującej się dziedziny badawczej. A. Błaszczak (Trad.). Tekst i dyskurs-text und diskurs, 8, 37-61. [Édition originale : Peter Lang, 2012].

Szymanek, K. (2012). Sztuka argumentacji. Ćwiczenia w badaniu argumentów. Warszawa : PWN.

Vigner, G. (1980). Une unité discursive restreinte : Le titre. Caractérisation et apprentissage. Le Français dans le monde, 156, 1-11...

Woods J. ; \& Walton, D. (1992). Critique de l'argumentation. Logique de sophismes ordinaires. M.-F. Antona et al. (Trads.). Paris : Éditions Kimé.

Wolny-Zmorzyński, K., et al. (2006). Prasowe gatunki dziennikarskie. Warszawa: Wydawnictwo Poltex.

Wojtak, M. (2015). Rozłożone gazety. Studia z zakresu prasowego dyskursu, języka i stylu. Lublin : Wydawnictwo Uniwersytetu Marii Curie-Skłodowskiej.

Zarefsky, D. (2019). The Practice of Argumentation. Effective Reasoning in Communication. Cambridge - New York : Cambridge University Press. 